\title{
Medaka fish provide new insights into osteoporosis
}

Phan, Q. T. et al. PNAS https://doi.org/10.1073/pnas.2006093117 (2020)

Continuous bone remodeling-a process by which bone resorption by osteoclasts is followed by bone formation by osteoblastsis essential to maintain bone structural integrity. An imbalance between cell activities, such as increased osteoclast activity, can result in reduced bone mass and bone disorders, including osteoporosis. The factors controlling the recruitment and activation of osteoclast and osteoblast progenitors to bone remodeling sites remain largely unknown, hampering the development of drugs to counteract bone loss.

In a new study published in PNAS, a team of investigators led by Christoph Winkler from the National University of Singapore used a medaka (Oryzias latipes) model to simulate osteoporosis and identify new mediators of osteoclast progenitor recruitment and differentiation.

The model has been previously described and consists of medaka expressing Rankl under the control of an inducible promoter.
Rankl encodes receptor-activator of NFk $\beta$ ligand (RANKL), a factor produced by osteoblasts to activate osteoclast progenitors; heat shock induction of transgenic Rankl expression in medaka larvae leads to ectopic differentiation of osteoclasts and excessive bone resorption, which simulates osteoporosis-like conditions.

Here, the investigators performed RNAseq analysis on osteoblast progenitors, premature osteoblasts and osteoclasts isolated from osteoporotic bone lesions of Rankl-induced medaka larvae. Transcriptome profiling revealed that upon Rankl induction, chemokine cxcl9-like (cxcl91, Olacxc19l) was up-regulated in osteoblast progenitors, whereas chemokine receptor gene $c x c r 3.2$ was up-regulated in osteoclasts.

Live imaging also revealed that upon Rankl induction, Cxcr3.2-positive macrophages are activated, migrate to bone matrix in the vertebrate column, and differentiate into osteoclasts, a process that was impaired in $c x c r 3.2$ mutants or when using antagonists AMG487 and NBI-74330 to chemically block Cxcr3.2 function. Altogether these results confirm the role of Cxcr3.2 in macrophage recruitment and osteoclast differentiation.

"Our in vivo experiments thus suggest that the chemokine-controlled process of osteoclast recruitment is druggable and that CXCR3 inhibitors can be used to modulate bone homeostasis. Targeting recruitment rather than activation of osteoclast progenitors opens new avenues for pharmacological treatment of a disease that affects millions of new patients every year and places extensive burden on families and health care systems," conclude the investigators in their report.

\section{Alexandra Le Bras}

Published online: 10 August 2020

https://doi.org/10.1038/s41684-020-0633-y

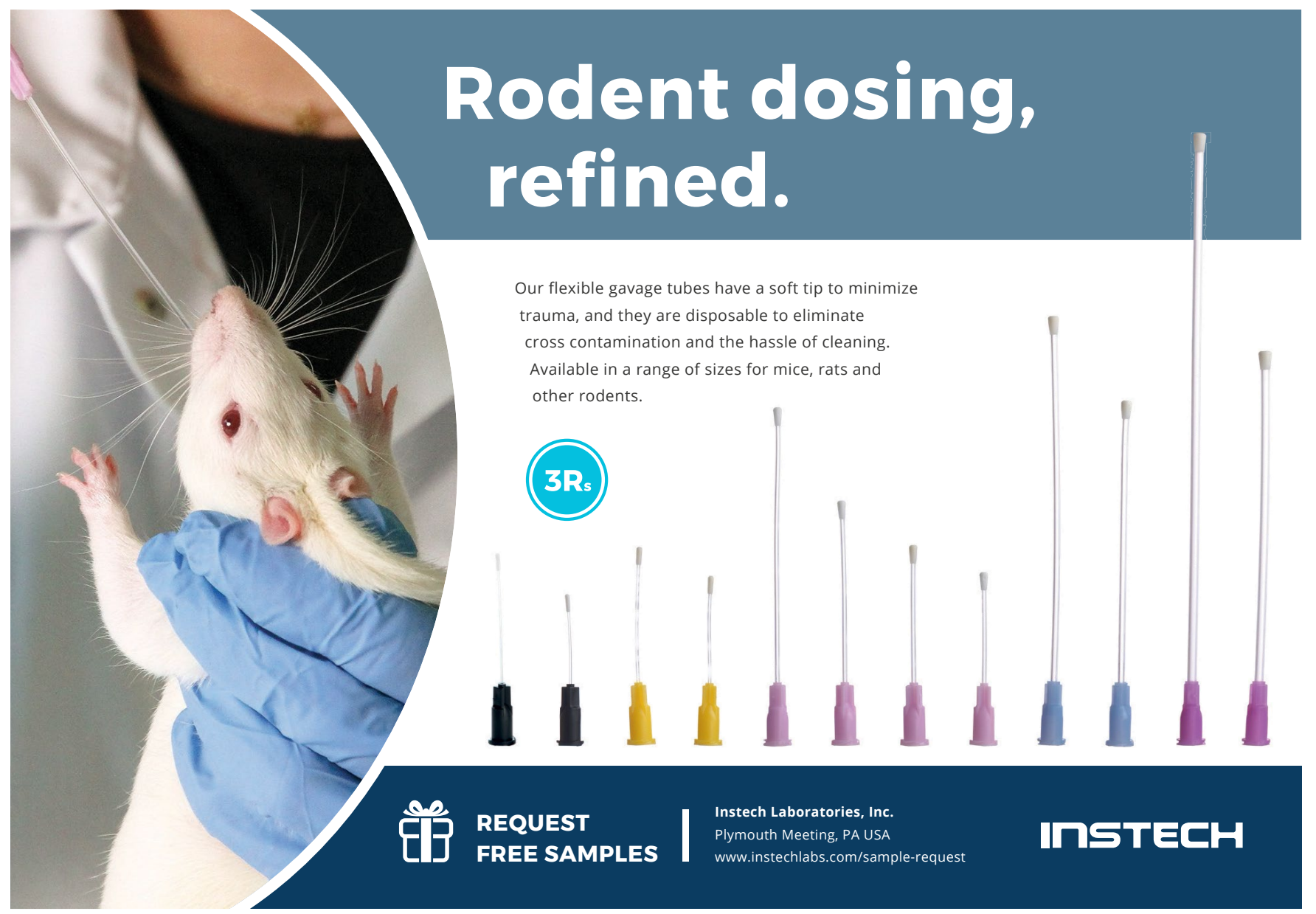

